\title{
Physiology
}

Aladjem, M. p14

Averbukh, Z. p14

Bandopadhayaya, G.P. p21

Barai, S. p21

Berman, S. p14

Bhaskaran, M. p92

Bhowmik, D. p21

Bichara, M. p77

Cohn, M. p14

Dellê, H. p82

Efrati, S. p14

Enomoto, A. p1

Falk, M.C. p35

Freund, R. p39

Frey, B.M. p51

Frey, F.J. p51

Fujihara, C.K. p82
Galle, J. p39

Galperin, E. p14

Gambaryan, S. p39

Gambhir, S. p21

Gopendro Singh, N. p21

Gupta, K.D. p21

Henderson, J.E. p72

Inoshita, S. p27

Karim, Z. p77

Kaye, M. p72

Konda, T. p1

Kumar, R. p21

Kuwahara, M. p27

Lote, C.J. p99

Malheiros, D.M.A.C. p82

Malhotra, A. p21

Mantovani, E. p82

Matsushita, J. p1
Mattar, A.L. p82

Mindel, G. p63

Modai, D. p14

Moriyama, T. p1

Morrison, A.R. p63

Noronha, I.L. p82

Okado, T. p27

Patel, C.D. p21

Radhakrishnan, N. p92

Raff, U. p39

Rapoport, M. p14

Rathi, M. p21

Reber, M. p39

Rodrigues, L.T. p82

Sasaki, S. p27

Schneider, R. p39

Schramm, L. p39

Schumacher, M. p51
Seibold, S. p39

Shirley, D.G. p99

Singhal, P.C. p92

Szutkowska, M. p77

Takahara, A. p1

Talaulikar, G.S. p35

Tanaka, H. p27

Terada, Y. p27

Vernimmen, C. p77

Vieira, J.M., Jr. p82

Vogt, B. p51

Vornberger, N. p39

Wanner, C. p39

Weissgarten, J. p14

Wrong, O. p72

Yu, Z. p51

Zatz, R. p82

\section{Subject Index Vol. 101, 2005}

Acid-base balance regulation p77

Acute renal failure p39

Aluminium excretion p99

- filterability p99

- reabsorption p99

Amlodipine p1

Ammonia synthesis p77

Analbuminemia p51

Angiotensin II p14, p77

- - type 1 receptor antagonism p14

Angiotensin-converting enzyme inhibitors p14

Apoptosis, mesangial cells p92

$L$-Arginine p39

Cell cycle p27

Chronic renal failure $\mathrm{p} 1$

Cilnidipine $\mathrm{p} 1$

Citrate p99

Distal renal tubular acidosis p72
Epithelial sodium channel p51

Gene expression regulation p77

Gitelman syndrome, pregnancy p35

Glomerular filtration rate $\mathrm{p} 21$

- - , reference range p21

Glucocorticoids p77

Heme oxygenase-1 p92

Hepatocyte growth factor $\mathrm{p} 92$

Hydrogen peroxide $\mathrm{p} 92$

Hypertension p1, p14, p63, p82

-, genetics p63

Hypokalaemia p35

Indian healthy adults, potential $\quad \mathrm{NH}_{4}{ }^{+}$urinary excretion $\mathrm{p} 77$ kidney donors $\mathrm{p} 21$

Interstitial fibrosis $\mathrm{p} 82$

Intrauterine imprinting, hypertension p63

Ischemia p27
Metabolic syndrome p1

Micropuncture study, aluminium reabsorption p99

Monocyte chemoattractant protein-1 p82

mRNA stability p77

Nagase analbuminemic rat p5 1

$\mathrm{Na}^{+}$homeostasis p63

- transporters p63

$\mathrm{Na}^{+}-\mathrm{K}^{+}\left(\mathrm{NH}^{+}\right)-2 \mathrm{Cl}^{-}$ cotransporter p77

Nedd4-2 protein expression p5 1

Nitric oxide $p 82$

- - synthases p39

N-type calcium channel blocker p1

Osteomalacia p72

Oxidative stress p92 p21 cyclin-cdk inhibitor p82

Pregnancy, Gitelman syndrome p35

Puromycin aminonucleoside p5 1

Renal failure, hypertension control p14

- function p63

- inflammation p82

- tubule p27

Serum/glucocorticoidinducible kinase p51

Simvastatin p82

Sodium diets, hypertension p14

Transforming growth factor- $\beta$ p82

Unilateral nephrotic syndrome p51

Vitamin D metabolites p72

\section{KARGER}

(c) 2005 S. Karger AG, Basel

Fax +4161306 1234 